\title{
Large eddy simulations in curved square ducts: variation of the curvature radius
}

\author{
C. MÜNCH and O. MÉTAIS
}

L.E.G.I. BP 53, 38041 Grenoble Cedex 09, France

\begin{abstract}
We present large-eddy simulations of the turbulent compressible flow at a low Mach number in curved ducts. The aim is to investigate the influence of the curvature radius $R_{c}$ on the flow. Three simulations are carried out at $R_{c}=3.5 D_{h}, 6.5 D_{h}$ and $10.5 D_{h}$ ( $D_{h}$ hydraulic diameter). We first validate our computations by comparison with the incompressible experiments performed by Chang et al. (1983, Turbulent flow in a strongly curved U-bend and downstream tangent of square cross-sections. Physico-chemical Hydrodynamics, 4(3), 243-269). We observe that the decrease of the curvature radius is accompanied by a strong intensification of the secondary transverse flows : a rise of $100 \%$ of the maximum of their intensity is obtained between the smaller and the higher values of $R_{c}$. We show that the secondary flows strength is directly related to the radial pressure gradient intensity. We observe a significant modification of the near-wall laws in the vicinity of each curved walls in correlation with the favourable or the adverse streamwise pressure gradient depending on the nature of the curvature. The influence of $R_{C}$ on the coherent vortices is also estimated.
\end{abstract}

\section{Introduction}

Turbulent flows on curved walls play an important role in many engineering applications such as turbines, heat exchangers or cooling channels of rocket engines. The study of the effects of a longitudinal curvature on turbulent flows has motivated numerous experimental and numerical works and several comprehensive reviews like the one by Bradshaw [13] and the more recent one by Patel and Sotiropoulos [12]. As pointed out by these authors, most of the studies have concentrated on the curvature effects on the concave or convex boundary layers in rectangular ducts of high-aspect ratio in attempts to simulate two-dimensional flow conditions. As far as numerical modelling is concerned, there have been considerable efforts to develop the statistical model able to correctly reproduce the curvature effects. As pointed out by Patel and Sotiropoulos [12], there is unfortunately no model suitable to properly take into account the curvature effects. The main difficulty arises from the three-dimensional nature of the curved turbulent flows. In a concave boundary layer, there is indeed evidence of longitudinal TaylorGörtler vortices. In closed duct of a small-aspect ratio, experiments have brought to light the development of an intense cross-stream flow due to the unbalance between the radial pressure gradient and the centrifugal forces, taking the shape of two large counter-rotating vortices of Ekman-type. This three-dimensional character has motivated several numerical works able to take into account the flow unsteadiness: curved boundary layers or curved channels have been studied by Moser and Moin [7], Lund and Moin [8], Silva Lopes and Piomelli [11]

${ }^{*}$ Corresponding author. E-mail: cecile.munch@hmg.inpg.fr 
using either direct numerical simulations (DNS) or (LES) large-eddy simulation. The threedimensional coherent vortices present in a curved duct of a large aspect ratio have recently been studied through unsteady Reynolds averaged Navier-Stokes (URANS) and Detached Eddy Simulations (DES) by Paik et al. [14].

Despite their numerous industrial applications, very few DNS or LES have been devoted to the study of the dynamics of the turbulent flow in curved ducts of the small aspect ratio. We have recently published several works, based on LES [18, 21, 23] investigating the three-dimensional nature of the coherent vortices in curved ducts. The ability for a largeeddy simulations technique to correctly represent the dynamics of curved flows was clearly demonstrated. The present paper completes these previous articles by varying the curvature radius to evaluate its influence on the flow statistics and on the coherent vortices. We focus on the case of ducts with a strong curvature $\left(D_{h} / R_{c} \geq 0.1\right)$. After a short description of the computational details in section 2 , section 3 presents the numerical results which are compared with the available experimental data issued from a flow in a U-bend provided by Chang et al. [1] and other measurements realized by Johnson [15] for a flow in a concave boundary layer. We specially investigate the influence of the curvature radius on the secondary flow, the velocity profiles and the turbulence structures. Finally, some concluding remarks are made in section 4.

\section{Characteristics of the simulations}

\section{$2.1 \quad$ Numerical methods}

The computer code used for our calculations solves the LES modified three-dimensional compressible Navier-Stokes equations in a curved square duct (see [16] for details). Previous applications of the code have been devoted to the study of turbulent compressible flows in straight ducts (see $[16,17])$. They observed a good agreement with previous incompressible DNS and LES for both statistical quantities and turbulent structures. Hébrard et al. [18] also initiated some simulations in the case of more complex geometries with this numerical code. The influence of the subgrid scales is modelled by the selective structure function model proposed by Lesieur and Metais [19]. In order to close the system of equations, we use three supplementary relations : (i) the Sutherland empirical law describing the variation of the molecular viscosity with the temperature. (ii) The equation of state describing the fluid as an ideal gas. (iii) The turbulent Prandtl number taken equal to 0.6. Curvilinear generalized coordinates are used (see [16] for details.) The discretization of the equations is made with the compact fully explicit McCormack scheme proposed by Kennedy and Carpenter [20] second order in time, fourth in space. The simulations are characterized by a Mach number equal to 0.5 and the bulk Reynolds number $\operatorname{Re}_{b}=6000$. These are based on the bulk velocity $\left(U_{b}\right)$, bulk density $\left(\rho_{b}\right)$ (see [16] for definitions), hydraulic diameter $\left(D_{h}\right)$ and the temperature at the walls $\left(T_{w}\right)$.

\subsection{Computational domain}

The computational domain is represented in figure 1. In our curvilinear coordinates system, $s$ designates the streamwise direction, $n$ the direction normal to the curved walls and $z$ the direction parallel to the curved walls. The various lengths are normalized by the hydraulic diameter $D_{h}$. The origin $O$ is taken at the inflow of the concave side (figure 1). The ducts have a square cross section. The angle of curvature is taken equal to $45^{\circ}$. We 


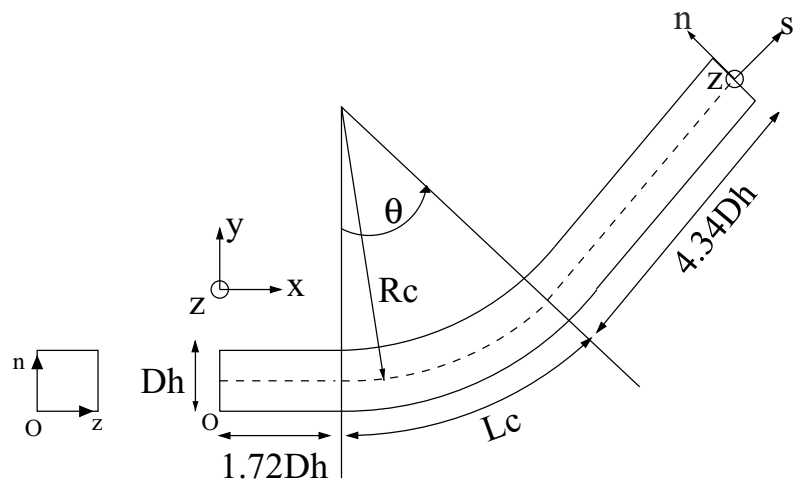

Figure 1. Geometry of computational domain.

consider three configurations with different mean curvature radius, $R_{c}=10.5 D_{h}, 6.5 D_{h}$ and 3.5 $D_{h}$. The different values for the length $L_{c}$ of the curved part and for the Dean number, $\mathrm{De}=\mathrm{Re}_{b} * \sqrt{\frac{D_{h}}{2 R_{c}}}$, are defined in table 1 . The computational domain is discretized using non-uniform numerical meshes in $s, n$ and $z$ directions. An hyperbolic-tangent stretching is applied in the transverse direction $n$ and $z$, the minimal and maximal grid spacing are shown in table 1. Another stretching in the $s$ direction is applied at the outflow to avoid the creation of spurious reflected waves. Note that we have performed grid independence test in previous studies (see [21]) and that the present grid resolution has proven to be sufficient.

\subsection{Boundary conditions}

The boundary conditions consist of a no-slip condition for the velocity and an uniform constant temperature $T_{w}$ imposed on the four walls. In the present computation, a fully developed turbulent flow is achieved at the curved duct inlet. Simultaneously, to the curved duct, a periodic (in the streamwise direction) straight square duct of sufficient length is simulated. It thus provides a turbulent field, at each time step, at the inlet of the curved duct. The inflow and outflow boundary conditions are obtained through the use of characteristics condition proposed by [22]. For further details, the readers can refer to [16].

\subsection{Definition of statistical quantities}

We define the mean quantities through time averaging, we note $\langle f(s, n, z)\rangle$ the mean value for any quantity $f(s, n, z, t)$. The mean components of the velocity: $\langle u\rangle,\langle v\rangle$ and $\langle w\rangle$ are noted

Table 1. Characteristics of the computational domain.

\begin{tabular}{lccc}
\hline Curvature radius & $10.5 D_{h}$ & $6.5 D_{h}$ & $3.5 D_{h}$ \\
$\frac{L_{c}}{D_{h}}$ & 8.64 & 5.50 & 3.14 \\
$\frac{L_{\text {total }}}{D_{h}}$ & 14.70 & 11.56 & 9.20 \\
De & 1310 & 1660 & 2270 \\
$\operatorname{Resolution}$ & $160 \times 50 \times 50$ & $128 \times 50 \times 50$ & $104 \times 50 \times 50$ \\
$n_{\min }^{+} / n_{\max }^{+}$ & $1.8 / 14.9$ & $1.8 / 14.9$ & $1.8 / 14.9$ \\
$\Delta x_{\min }^{+} / \Delta x_{\max }^{+}$ & $2.5 / 39$ & $2.6 / 40$ & $2.6 / 40$ \\
\hline
\end{tabular}


as $U, V$, and $W$. The root mean square velocities are noted as $u_{\mathrm{rms}}, v_{\mathrm{rms}}, w_{\mathrm{rms}}$ and $\left\langle u^{\prime} v^{\prime}>\right.$ is one of the Reynolds stress components. The normalization of those quantities is realized by either the bulk or the local friction velocities. The bulk quantity $f_{b}$ corresponds to $\langle f\rangle$ integrated along the two transverse coordinates. The bulk velocity $U_{b}$ involves the density and is defined as

$$
U_{b}=[\rho U]_{b} / \rho_{b}
$$

The friction velocities $U_{\tau_{c c}}$ and $U_{\tau_{c v}}$ are based on the wall shear stresses $\tau_{c c}$ and $\tau_{c v}$, respectively, on the concave and the convex walls. We designate as $n^{+}$the distance, from the considered wall, normalized by the local viscous thicknesses. We use the fact that the plane $z / D_{h}=0.5$ is a symmetry plane in the representation of the results.

\section{Results}

\subsection{Validation}

In this part, we discuss the influence of the curvature on the mean velocity components and compare our results with the experiments. We first consider the turbulent flow for the duct with the strongest curvature $\left(R_{c}=3.5 D_{h}\right)$. Detailed experiments were conducted by Chang et al. [1] for an incompressible flow in a $180^{\circ}$ curved duct of square cross section and a curvature radius equal to 3.35 times the hydraulic diameter. The mean flow and turbulence characteristics were determined for a Dean number De $=2.1 \times 10^{4}$ and a Reynolds number equal to $5.6 \times 10^{4}$, which are ten times higher than our values. A straight inlet of $31 D_{h}$ was placed upstream of the bend to obtain a fully developed turbulent flow. This inlet condition is comparable with our inlet periodic duct. It is important to point out that the experimental parameters (Reynolds number, Dean number, angle of curvature) are very distinct from our numerical parameters. Despite these differences, the following results indicate that we observe not only a good qualitative agreement but also a satisfactory quantitative agreement. Although we consider a compressible case, $M_{a}=0.5$, the compressibility effects are very weak. To demonstrate this assumption, we provide the results obtained for the same flow configuration but with a Mach number equal to 0.35 . This constitutes the lowest limit which can be reached with our numerical code which is conceived for compressible flow and which uses explicit numerical methods. Figures 2, 4 and 5 show the small difference between the two simulations at different Mach numbers and justify our validation with an incompressible case. Different profiles are plotted as a function of the distance $n / D_{h}$ from the concave wall, for fixed values of $z / D_{h}$ and $s / D_{h}$ or $\theta$. In figure 2, the streamwise mean velocity profiles are plotted in the symmetry plane for $\theta=0^{\circ}, 3^{\circ}, 45^{\circ}$. At the inlet of the curved part $\left(\theta=0^{\circ}\right)$, the variables are normalized by the centreline velocity at the inflow.

Figure 2(a) shows that, at the inflow, the profile is symmetric with respect to the plane $n / D_{h}=0.5$ since the curvature effects are not yet effective. At $\theta=3^{\circ}$, a slight asymmetry of the profiles can be distinguished: the velocity becomes larger close to the convex wall $\left(n / D_{h}=1\right)$ than on the opposite wall. At $\theta=45^{\circ}$, the opposite effect arises: the flow is now slowed down near the convex side. As far as figures 2(a) and (b) are concerned, our results fit quite well with the experiments. One may however note, in figure 2(b), that the experimental data exhibit higher velocities in the vicinity of each wall: this can be explained by the higher value of the Reynolds number. In figure 2 (c), we obtain a smaller intensity of the streamwise velocity close to the convex wall as compared with the experiment, certainly due to the Reynolds number effects. 
a)

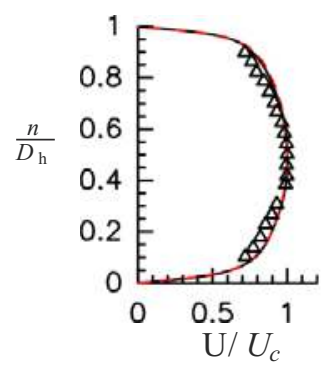

b)

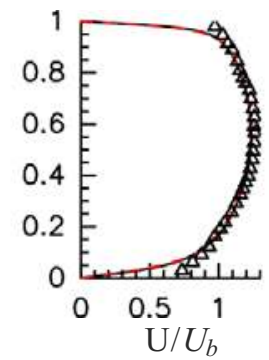

c) $\quad 45^{\circ}$

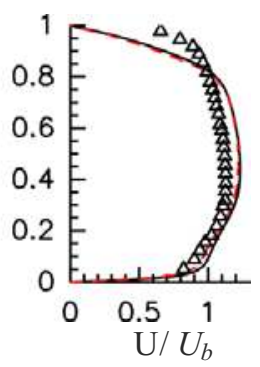

Figure 2. Streamwise mean velocity in the symmetry plane, Chang $\triangle, R_{c}=3.5 D_{h}-M_{a}=0.5 D_{h}$ and - $M_{a}=0.35$ for $\theta=$ (a) $0^{\circ}$, (b) $3^{\circ}$, and (c) $45^{\circ}$.

To interpret the various modifications of the velocity profiles induced by the curvature effects, we next consider the streamwise pressure gradient which is plotted on figure 3 . In the straight inlet part of the duct until the beginning of the curvature, the boundary layer is subjected to a favourable pressure gradient on the convex side which induces the increase of the streamwise velocity component on the inner wall, as noted by Chang et al. and Kim et al. $[1,2]$. On the opposite wall, an adverse streamwise pressure gradient arises explaining the slight diminution of the $U / U_{b}$. Further downstream of the curved part, the behaviour of the pressure changes: the adverse pressure gradient is now observed near the convex wall until the end of the bend and is accompanied by velocity decrease in that region. The situation is reversed near the concave wall.

Figure 3 also shows that an important radial pressure gradient takes place within the duct: it leads to the appearance of a strong secondary motion as observed in previous laboratory experiments [1,3] and in our previous numerical works [23]. The secondary flow is defined as the flow perpendicular to the main flow, which itself follows the curvilinear abscissa. Figure 4 compares the LES results and the experimental data for the velocity component $V / U_{b}$ normal to the curved walls: the profile functions of the normal coordinate $n / D_{h}$ are plotted in the duct section located at $\theta=45^{\circ}$ and for three values of $z / D_{h}$. Again, the influence of the Mach number is shown to be small.

For the three $z$ locations, $V / U_{b}$ exhibits negative values: this indicates that the fluid is flowing from the convex towards the concave wall near the duct centre. It will be shown in section 3.2 that this central current is compensated by a strong current from the concave wall

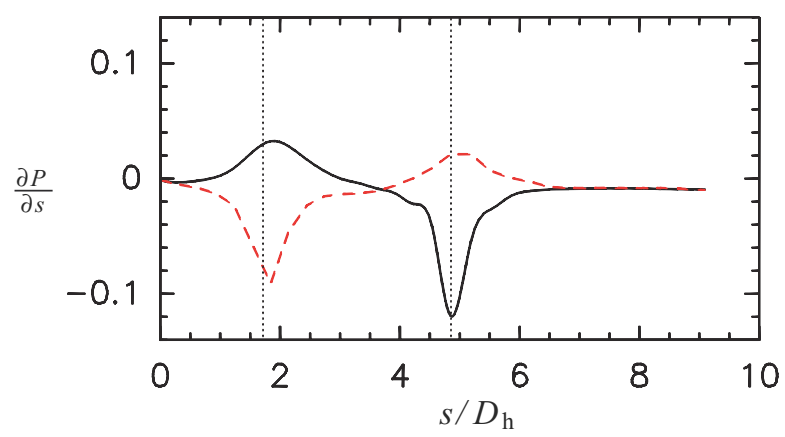

Figure 3. Streamwise pressure gradient in the case $R_{c}=3.5 D_{h}$ on - concave wall, - - convex wall. The vertical lines correspond to the beginning and the end of the curved parts of the duct. 
a) $z / D_{\mathrm{h}}=0.5$

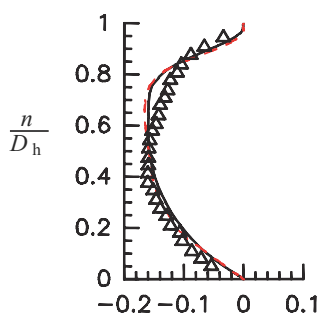

b) $z / D_{\mathrm{h}}=0.35$

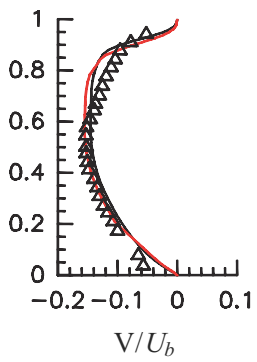

c) $z / D_{\mathrm{h}}=0.25$

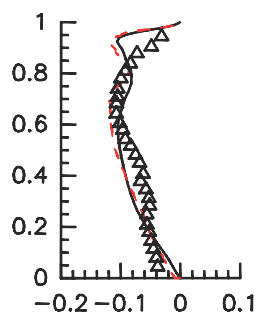

Figure 4. Profiles as a function of $n$ of the normal component $V$ of the mean velocity: Chang $\triangle, R_{c}=3.5 D_{h}-$ $M_{a}=0.5--M_{a}=0.35$, for $z / D_{h}=$ (a) 0.5 , (b) 0.35 and (c) 0.25 .

towards the convex wall localized in the vicinity of the duct lateral walls (see figures 10-12). The highest values of $V / U_{b}$ appear in the symmetry plane $\left(z / D_{h}=0.5\right)$, reaching almost $20 \%$ of the bulk velocity. For the smaller values of $z / D_{h},\left|V / U_{b}\right|$ decreases progressively. A good agreement is obtained between our LES and the experiment of Chang et al. [1] demonstrating the ability for the LES to correctly reproduce the secondary flows. As explained by [23], this secondary flow originates from the unbalance between the radial pressure gradient (see figure 8 ) and the centrifugal forces. Close to the sidewalls, the radial pressure gradient drives the fluid from the concave towards the convex wall since the velocity and thus the centrifugal forces tend to zero. In the core region, the mass conservation requires a flow in the opposite direction.

In figure 5, the Reynolds shear stress component $\left\langle u^{\prime} v^{\prime}\right\rangle$ is plotted at two stations: $\theta=3^{\circ}$ and $45^{\circ}$, as a function of $n / D_{h}$ in the symmetry plane and closer to the sidewalls. In our simulation, we observe the effects of curvature from $\theta=3^{\circ}:\left\langle u^{\prime} v^{\prime}\right\rangle$ is suppressed close to the convex wall and amplified near the concave wall. This amplification goes on as we move downstream: at $45^{\circ},\left\langle u^{\prime} v^{\prime}\right\rangle$ indeed reaches more than $6 \%$ of $U_{b}^{2}$ near the concave wall.

Although a similar behaviour is observed in the computation and the experiment, the LES yields an overestimation of the Reynolds stress for $\theta=3^{\circ}$ close to the concave wall. At $\theta=45^{\circ}$, the agreement is better. The discrepancy for $\theta=3^{\circ}$ can be explained by the Reynolds number which is weaker in our case, therefore the intensity of the Reynolds stress component could be different. At the end of the curved part, the curvature effect dominates the Reynolds number effect and the curves exhibit very close values. The results shown in figure 5 bear out

a) $z / D_{h}=0.5$

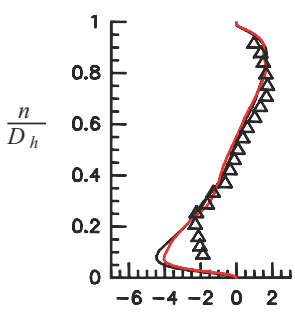

b) $z / D_{h}=0.25$

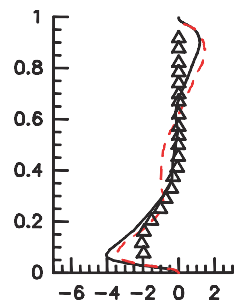

c) $z / D_{h}=0.5$

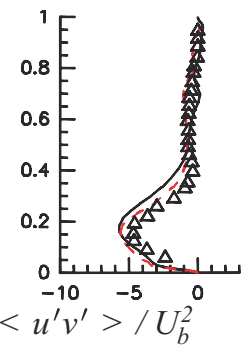

d) $z / D_{h}=0.25$

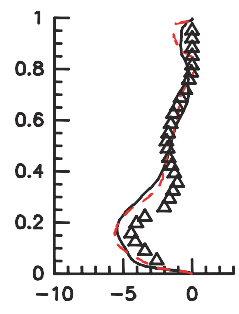

Figure 5. Reynolds stress component $\left\langle u^{\prime} v^{\prime}\right\rangle$ Chang $\triangle, R_{c}=3.5 D_{h}-M_{a}=0.5--M_{a}=0.35$ for $\theta=3^{\circ}$, $z / D_{h}=$ (a) 0.5 , (b) 0.25 and for $\theta=45^{\circ}, z / D_{h}=$ (c) 0.5 , (d) 0.25 . 


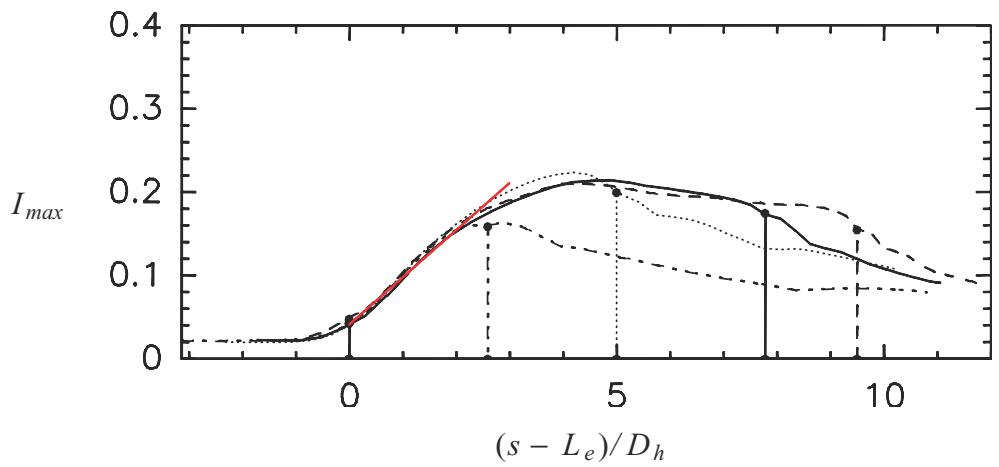

Figure 6. Maximum value of the secondary flow intensity in curved ducts with $R_{c}=10.5 D_{h}$ and an angle of curvature equal to - - - $15^{\circ}, \ldots \ldots 30^{\circ},-45^{\circ}$ and $---60^{\circ}$. The rougeoblique line is the linear function given by formula two. The vertical lines correspond to the curved parts.

the stabilizing effect of the convex side and the production of turbulence on the concave wall found by many experiments (see $[1,2,4]$ ). Note again the very small influence of the Mach number.

\subsection{Influence of the curvature radius}

3.2.1 Mean quantities. We here investigate the role played by the curvature radius by comparing the simulations with three different values of $R_{c}: 10.5,6.5$ and $3.5 D_{h}$. As previously mentioned, the curvature angle for the three cases is taken equal to $45^{\circ}$. This choice originates from a prefatory study aimed at the determination of the influence of the curvature angle for a fixed value of the curvature radius equal to $10.5 D_{h}$. We carried out simulations for four cases: $15^{\circ}, 30^{\circ}, 45^{\circ}$ and $60^{\circ}$. In figure 6 , we represent the maximum of the secondary flow intensity, defined as $I_{\max }=\sqrt{\frac{V^{2}+W^{2}}{U_{b}^{2}}}$, for the four cases. $I_{\max }$ is plotted as a function of $\left(s-L_{e}\right) / D_{h}$, where $L_{e}$ is the straight inflow length, so that the origin of the abscissa axis corresponds to the beginning of the curvature.

In the straight inflow, the secondary flow has an intensity of $2 \%$ of the bulk velocity which is the characteristic of Prandtl's secondary flow of second kind which develops in the corner of a straight duct (see [16]). From the beginning of the curvature, we observe that the intensity of the secondary flow for the four cases increases with the same rate until the end of each respective curved part. At the beginning of the curvature, this growth is quasi-linear with

$$
I_{\max }=A\left(s-L_{e}\right) / D_{h}+B,
$$

with $A=0.055$ and $B=0.041$ (plotted in figure 6). In the case where the angle is equal to $15^{\circ}$, the maximum is reached at the end of the curved part and is equal to $15 \%$. For the three other cases, the maxima are identical, approximatively equal to $22 \%$ of the bulk velocity, the peak values being reached before the end of the curved part. In the straight outlet part, $I_{\max }$ decreases for the four cases. This result brings out the existence of a limit value for $I_{\max }$ of $22 \%$ for this value of $R_{c}$. It is important to note that the comparison between the $45^{\circ}$ and $60^{\circ}$ cases indicates that $I_{\max }$ is independent of $\theta$ in the curved part for a sufficiently high angle. This justifies the choice of an angle of $45^{\circ}$ in the next simulations: we indeed estimate that this angle is sufficient to allow the full development of the intense secondary flows associated with the curvature. The agreement with the experimental measurements presented in the previous section corroborates this observation. 


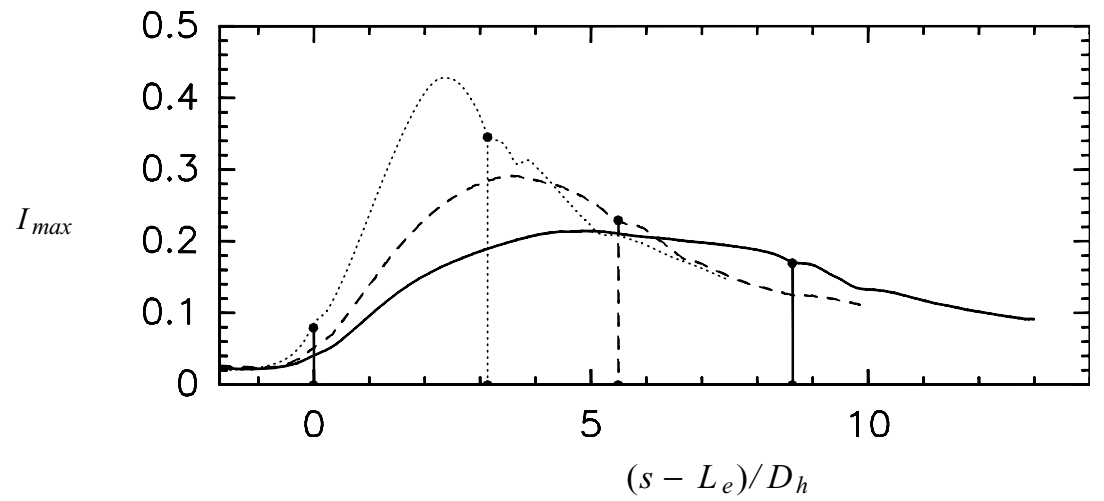

Figure 7. Maximum of the secondary flow intensity $I_{\max }:-R_{c}=10.5 D_{h},--R_{c}=6.5 D_{h}, \cdots R_{c}=3.5$ $D_{h}$. The vertical lines correspond to the limits of the curved parts.

We now investigate the influence of the curvature radius on the secondary flow intensity. In figure $7, I_{\max }$ is plotted as a function of $\left(s-L_{e}\right) / D_{h}$ with $L_{e}=1.7 D_{h}$ for the three cases with different $R_{c}$ and a fixed value of the angle $\theta=45^{\circ}$. We observe a similar behaviour as in figure 6: at the inflow, $I_{\max }$ is approximatively equal to $2 \%$ of the bulk velocity; in the curved parts the intensity increases and declines at the outflow. However, when the curvature radius changes, the growth rate in the curved part is also modified: the smaller value of $R_{c}$ corresponds to the faster growth. The peak for $R_{c}=3.5 D_{h}$ exceeds $40 \%$ of the bulk velocity, $30 \%$ for $R_{c}=6.5 D_{h}$ and $20 \%$ for $R_{c}=10.5 D_{h}$.

As explained above, the secondary flow is generated by the pressure difference between the two curved wall: we represent $C_{p}=\left(p-p_{i}\right) / \rho U_{b}^{2}$ in figure 8 , where $p_{i}$ is the pressure at the duct inlet.

In the three ducts, $C_{p}$ rises on the concave wall and decreases on the convex side. It generates a pressure difference between the two curved walls whose intensity is almost constant in the curved part. This phenomenon starts before the beginning of the curvature and fades away after its end. When the curvature radius becomes weaker, the difference between the two values of $C_{p}$ increases, which means a stronger radial pressure gradient than in the case $R_{c}=10.5 D_{h}$. Since the radial pressure gradient drives the fluid from the concave to the convex wall close to the sidewalls, this secondary transverse flow is therefore enhanced. This result explains the

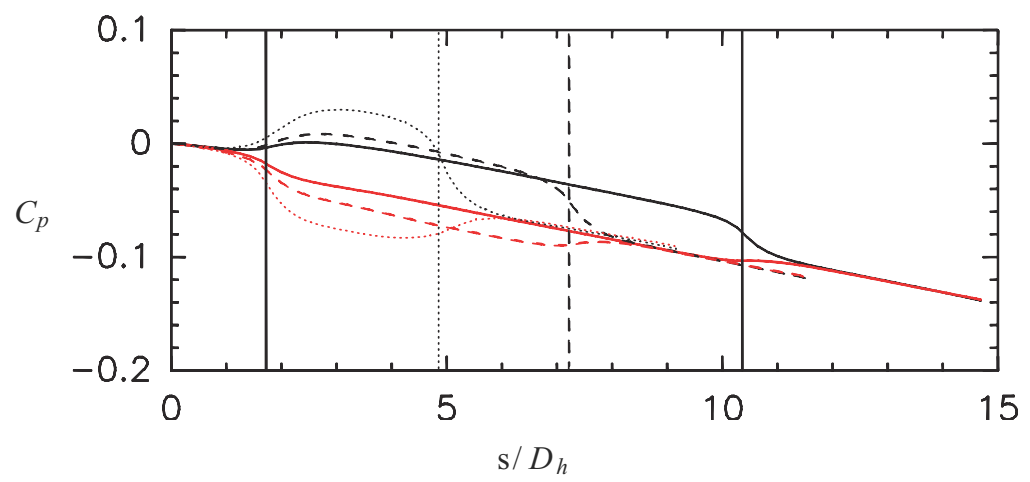

Figure 8. $C_{p}$ on the concave and on the rougeconvex wall: $-R_{c}=10.5 D_{h},--R_{c}=6.5 D_{h}, \cdots R_{c}=3.5$ $D_{h}$. The vertical lines correspond to the limits of the curved parts. 


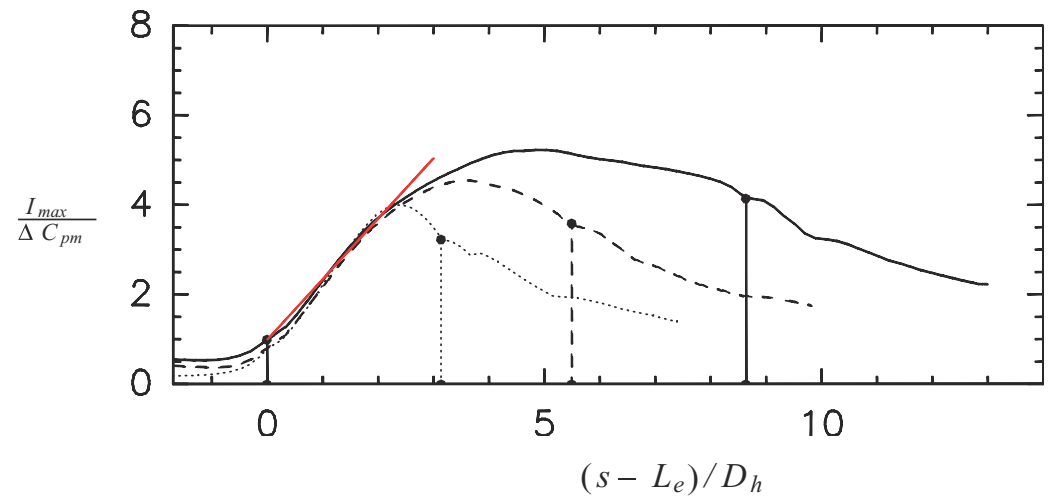

Figure 9. Normalized maximum of the secondary flow intensity $I_{\max } / \Delta C_{p m}$ : $-R_{c}=10.5 D_{h},---R_{c}=6.5$ $D_{h}, \cdots \cdots R_{c}=3.5 D_{h}$. The rougeoblique line is the linear function given by formula (3). The vertical lines correspond to the limits of the curved parts.

different rates of growth of $I_{\max }$. Since $I_{\max }$ and $C_{p}$ are interconnected, we next normalize $I_{\max }$ by the maximum difference between the values of $C_{p}$ on the two curved wall: $\Delta C_{p m}$. In figure 9, we plot $I_{\max } / \Delta C_{p m}$ as a function of $\left(s-L_{e}\right) / D_{h}$ in the three cases.

We observe that this normalization leads to the same rate of growth for $I_{\max } / \Delta C_{p m}$ for the three values of $R_{c}$. The linear law, which describes the increase of $I_{\max }$ in the curved part, is thus only dependent on the radial pressure gradient between the two curved walls. In our case, we find a law

$$
\left.I_{\max }=\Delta C_{p m} *\left(A\left(s-L_{e}\right) / D_{h}+B\right)\right),
$$

with $A=1.35$ and $B=1.0$ (plotted in figure 9). In fact, this law is similar to the first found in figure 6 since for $R_{c}=10.5 D_{h}, \Delta C_{p m}=0.041$. We note that, with this normalization, the peak is higher for the higher value of the curvature radius, since the length of the curved part is longer in that case.

In the next figures, we show the mean secondary flow in half cross-sections. We compare our results in three sections: at the beginning of the curvature $\left(0^{\circ}\right)$, at $\theta=30^{\circ}$ and at the outflow. As mentioned above, in a straight duct, another type of secondary flow develops due to the corner, usually called the Prandtl's secondary flow of second kind. They generate eight counter rotating cells, two in each corner (see [16]). At the beginning of the curvature, they are still perceptible in figures 10 (b) and (c) for $R_{c}=6.5$ and $10.5 D_{h}$. For the smallest value of $R_{c}$, the pressure gradient between the concave and convex walls due to the curvature is the most important. Due to the non-locality of the pressure effects, these can be filled upstream of the curved part as notable on the $C_{p}$ coefficient was shown in figure 8. Consequently, an intense stream close to the side wall is already apparent right at the beginning of the curved $\operatorname{part}\left(\theta=0^{\circ}\right.$, figure 10a)). At the station $\theta=30^{\circ}$, the secondary flow exhibits the same pattern for the three values of $R_{c}$. The flow is driven from the concave to the convex wall along the sidewall, then from the sidewall to the middle of the convex wall, along the convex wall and finally towards the core region in the symmetry plane. The resulting flow organisation is a cell close to the convex wall, called the Ekman cell (as denominated by Mees et al. [5]). We observe that the cell is more confined near the duct corner for the smaller values of $R_{c}$. Furthermore, the intensity of the secondary flow is higher in the case of smaller curvature radius.

In figure 12, the Ekman cells are now totally developed and consist of pairs of large longitudinal vortices which occupy the full width of the duct. Since the secondary flows grow in intensity when $R_{c}$ decreases, the Ekman cells are larger in that case inducing a translation of 

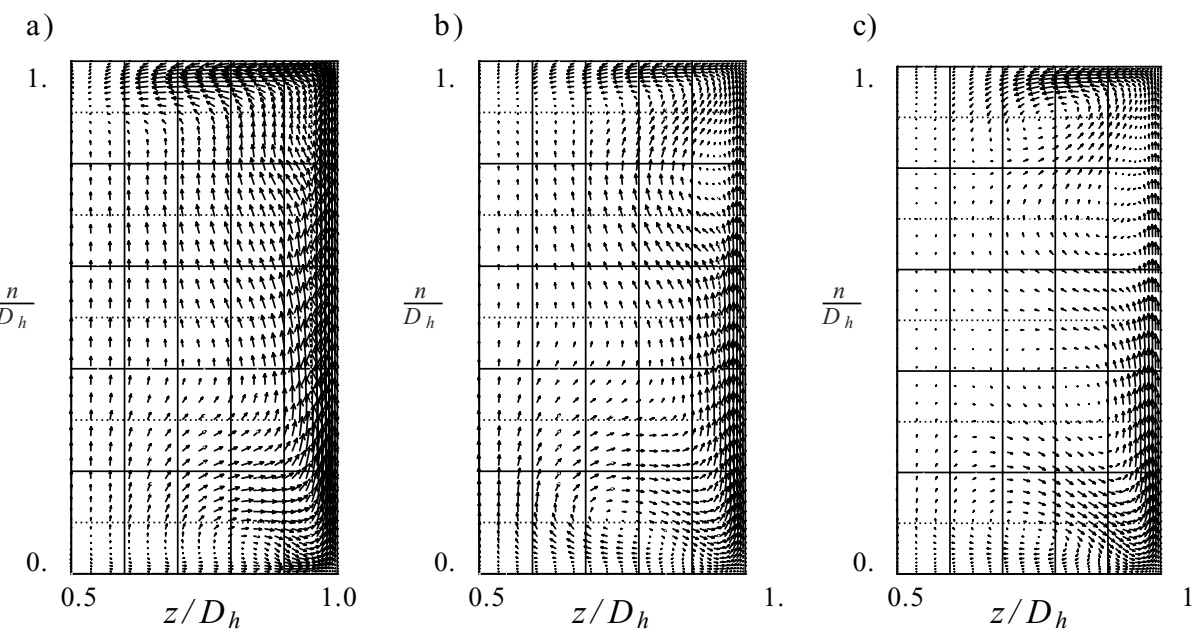

Figure 10. Half cross-sections showing the secondary flow at the beginning of the curved part, (a) $R_{c}=3.5 D_{h}$, (b) $R_{c}=6.5 D_{h}$ and (c) $R_{c}=10.5 D_{h}$.

their centre towards the duct core: for $R_{c}=10.5 D_{h}$, the centre is located at $n / D_{h}=0.8$ when it is placed at $n / D_{h}=0.7$ for $R_{c}=3.5 D_{h}$. We also note the stronger value of the vertical velocity in the symmetry plane for smaller values of the curvature radius.

To quantify the intensity of the secondary flow, we plot in figure 13 the velocity component $V / U_{b}$, normal to the curved wall, as a function of the normal coordinate $n / D_{h}$ in the duct symmetry plane $\left(z / D_{h}=0.5\right)$ and for three different cross-sections. In the straight inflow part, $V / U_{b}$ is very weak since the secondary cells of Prandtl's second remain located at the duct corners. Figure 13(b) shows a significant amplification of the secondary flows as one moves further downstream. $V / U_{b}$ indeed exhibits negative values indicating the development of the secondary flow going from the convex to the concave side. At this downstream location, the secondary flow amplitude is weakly influenced by the value of the curvature radius. This

a)

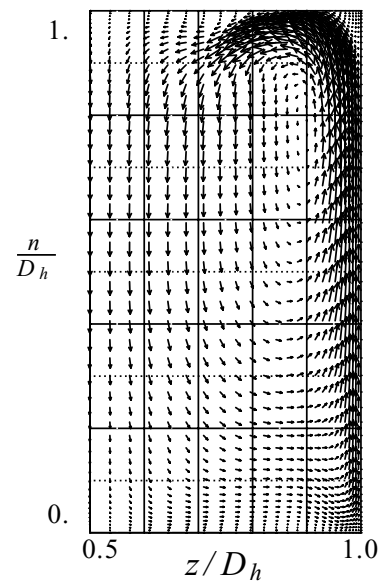

b)

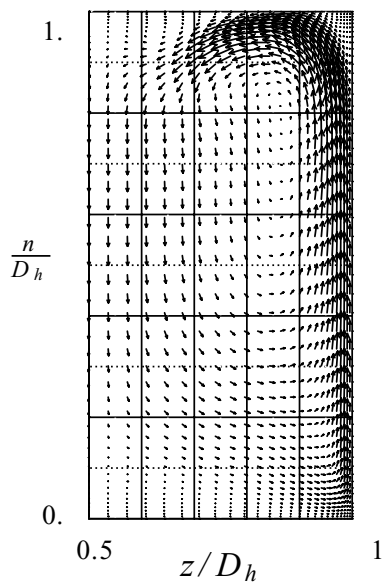

c)

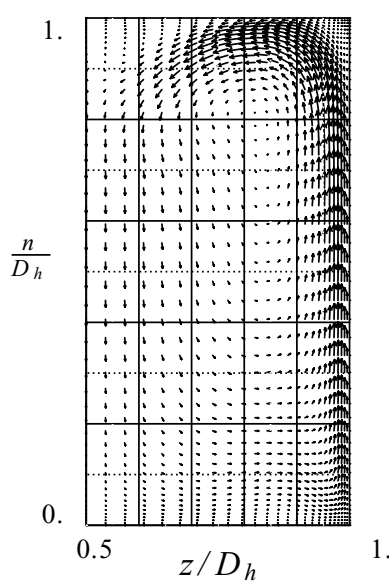

Figure 11. Half cross-sections showing the secondary flow at $\theta=30^{\circ}$ (a) $R_{c}=3.5 D_{h}$, (b) $R_{c}=6.5 D_{h}$ and (c) $R_{c}=10.5 D_{h}$ 
a)

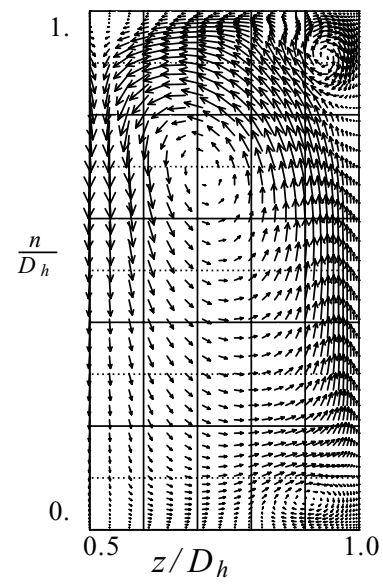

b)

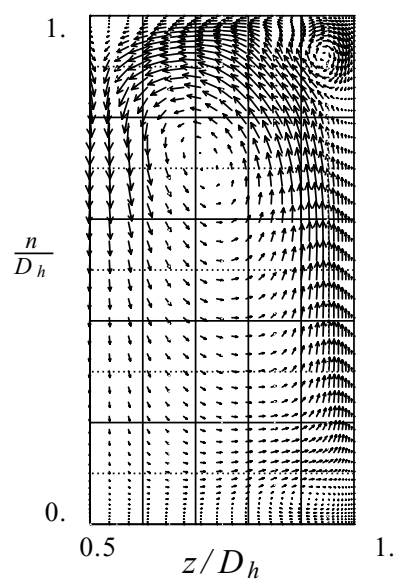

c)

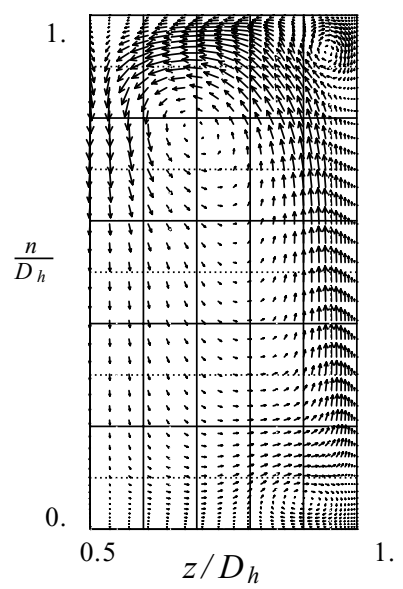

Figure 12. Half cross-sections showing the secondary flow at the outflow (a) $R_{c}=3.5 D_{h}$, (b) $R_{c}=6.5 D_{h}$ and (c) $R_{c}=10.5 D_{h}$.

influence is much greater near the end of the curved part (figure 13(c)) where it can be clearly seen that the secondary flow is more and more enhanced when the curvature radius is decreased.

Now, we focus our attention on the behaviour of the mean velocity profiles in the curved boundary layer and in particular the near-wall behaviour. For modelling purposes wall functions are often used to mimic the near-wall region. Well-defined wall laws remain uncertain for the boundary layers submitted to the curvature effects. Experimental and numerical studies $([7,9,15,24])$ have shown that the longitudinal velocity profiles on the concave wall lie below the classical logarithmic law defined as $u^{+}=\frac{1}{\kappa} \ln \left(y^{+}\right)+5.5$, with $\kappa=0.418$ being the Karman constant while the velocity profiles develop above this law close to the convex wall. Furthermore, Piomelli et al. [25] have shown that the deviation from the standard log-law is directly linked to the intensity of the longitudinal pressure gradient: a strong favourable pressure gradient is associated with a strong acceleration of the boundary layer and with a logarithmic behaviour for the streamwise mean velocity well above the standard log-law.

In figure 14, we plot the streamwise pressure gradient, similarly to figure 3 but for the three values of $R_{c}$, in black on the concave wall and in red on the opposite wall. We take the abscissa to be the inclination angle to more easily compare the three different curvatures. We
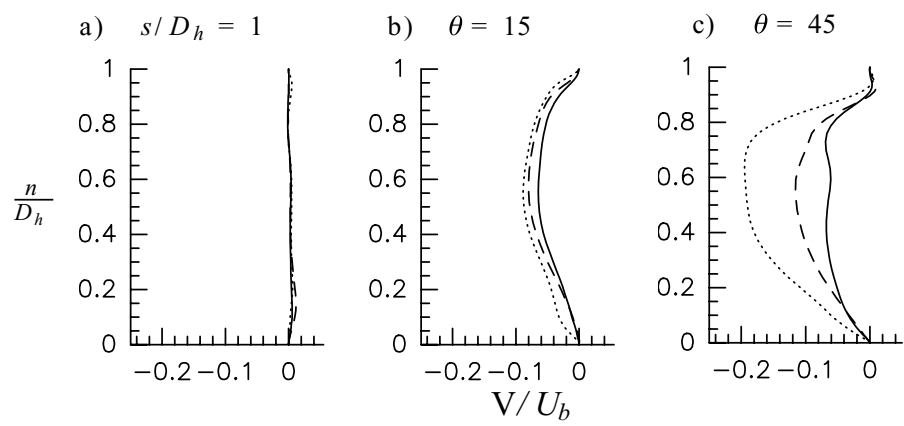

Figure 13. Profiles as a function of $n$ of the normal component $V$ of the mean velocity: $-R_{c}=10.5 D_{h},---$ $R_{c}=6.5 D_{h}$ and $\cdots \cdots R_{c}=3.5 D_{h}$ for (a) $s / D_{h}=1$, (b) $\theta=15$ and (c) $\theta=45$. 


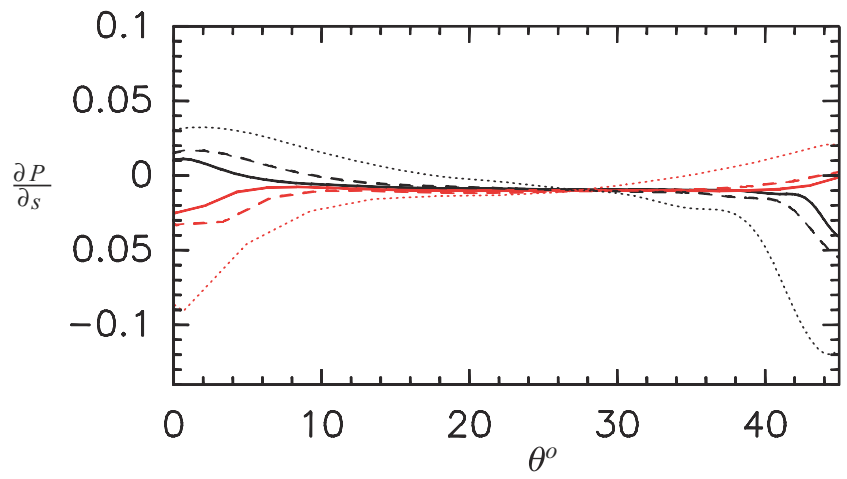

Figure 14. Streamwise pressure gradient as a function of the angle on the concave and the convex wall for $R_{c}=10.5 D_{h},--R_{c}=6.5 D_{h}$ and $\cdots . . R_{c}=3.5 D_{h}$.

observe that the streamwise pressure gradient, at the beginning of the curvature, is positive on the concave wall corresponding with an adverse pressure gradient. It is negative on the opposite wall indicating a favourable pressure gradient. The pressure gradient changes the sign further downstream and becomes strongly negative on the concave side and slightly positive on the convex side. The amplitude of the pressure gradient is directly linked with the curvature magnitude since it is far more important when the curvature radius is small. The two boundary layers on each of the curved walls therefore evolve in the presence of a favourable or an adverse pressure gradient. The dynamics of both boundary layers is however made more complex due to the presence of the Ekman cells located near the convex side of the duct. If one considers the duct symmetry plane, the flow ejection on the convex wall will be associated with a decrease of the friction velocity on this wall, while the flow impact on the opposite wall yields an increase of the local friction velocity. These modifications directly impact the behaviour of $u^{+}$since it is normalized by the friction velocity. These effects will be more and more important when one moves further downstream since the cells intensity increases.

In figure 15, we represent the mean velocity on each wall (concave and convex) in local wall coordinates: $U^{+}$and the normalized distance from the considered wall $n^{+}$are based on the local value of the friction velocity $U_{\tau_{c c}}$ (for the concave wall) or $U_{\tau_{c v}}$ (for the convex wall). The profiles are plotted in the symmetry plane at four stations of the duct: at the inlet, for $\theta=15^{\circ}, 30^{\circ}$ and $45^{\circ}$, in black close to the concave wall, in blue close to the convex one.

We compare our velocity profiles on the concave wall with those obtained in the experiment performed by Johnson [15] in a fully developed boundary layer submitted to destabilizing the concave curvature. In the experiment, the Reynolds number based on the momentum thickness and the free-stream velocity, $\mathrm{Re}_{\theta}$, is equal to 1450 . The curvature radius, $R_{c}$, is equal to $136 \mathrm{~cm}$ and the boundary layer thickness to approximatively $\delta=10 \mathrm{~cm}$, thus $R_{c} / 2 \delta=6.8$. The experimental data consist of two different sets: with $\Delta$ or without $\Delta$ inflow grid-generated turbulence. For comparison, we plot the log-law observed on a flat wall in a duct (see [26]): $U^{+}=3.2 \ln \left(n^{+}\right)+3.9$ which takes into account the influence of the lateral wall on the velocity profiles.

In figure 15(a), we check that the mean streamwise velocity profiles follow the log-law expected in a straight duct: this constitutes a validation of our LES. In three figures 15(b), (c) and (d), we add the experimental data from Johnson [15]. For $\theta=15^{\circ}$, figure 15 (b), our profiles separate from the log-law corresponding to a flat wall. Close to the concave side, the velocity profiles develop under the law while they go over it close to the opposite curved wall: this is consistent with the previous experimental observations in curved concave and convex 
a)

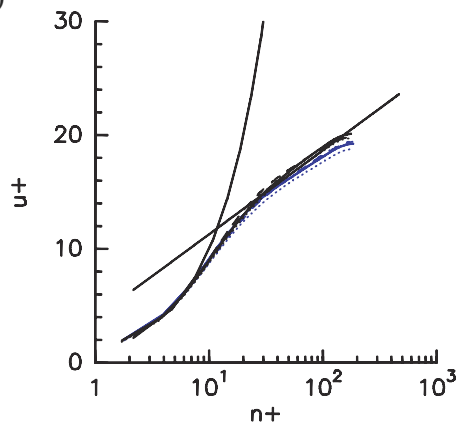

c)

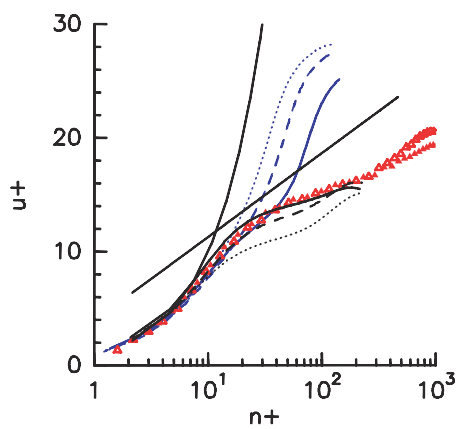

b)

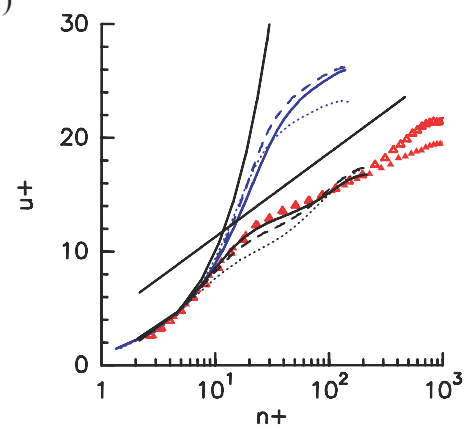

d)

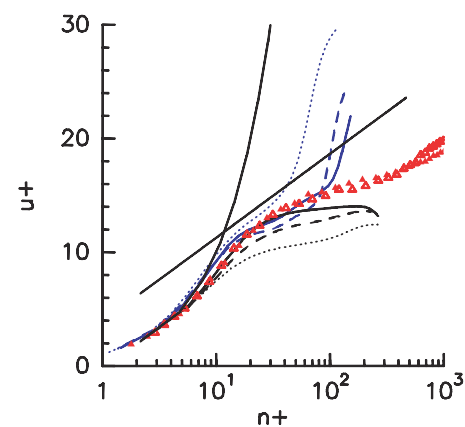

Figure 15. $U^{+}$as a function of $n^{+}$in semi-log graphics, close to the concave and the convex walls: $-R_{c}=10.5 D_{h}$, - - $R_{c}=6.5 D_{h}$ and $\cdots . . R_{c}=3.5 D_{h}$, in the concave boundary layer [15] $\triangle$, $\mathbf{\Delta}$. (a) At the inlet of our duct, for $\theta=$ (b) $15^{\circ}$ and (c) $30^{\circ}$ and (d) $45^{\circ}$.

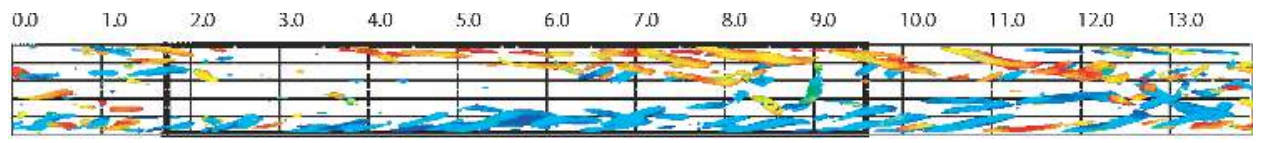

Figure 16. Instantaneous isosurfaces of $Q=0.7 U_{b}^{2} / D_{h}^{2}$, colored by the longitudinal vorticity, close to the convex wall: $R_{c}=10.5 D_{h}$, see http://www.informaworld.com/mpp/uploads/jot-2005-0072-file001.mpg

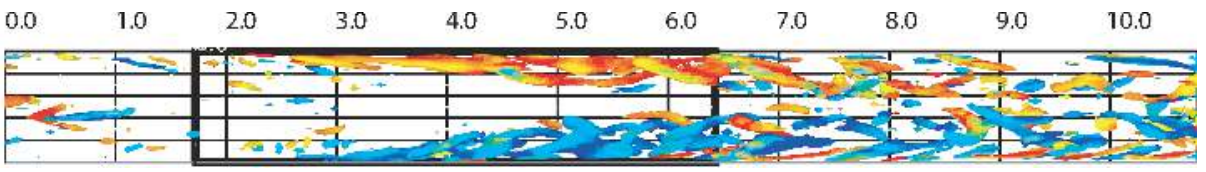

Figure 17. Instantaneous isosurfaces of $Q=0.7 U_{b}^{2} / D_{h}^{2}$, colored by the longitudinal vorticity, close to the convex wall: $R_{c}=6.5 D_{h}$, see http://www.informaworld.com/mpp/uploads/jot-2005-0072-file002.mpg

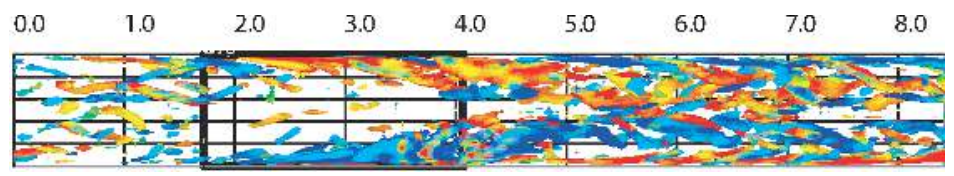

Figure 18. Instantaneous isosurfaces of $Q=0.7 U_{b}^{2} / D_{h}^{2}$, colored by the longitudinal vorticity, close to the convex wall: $R_{c}=3.5 D_{h}$, see http://www.informaworld.com/mpp/uploads/jot-2005-0072-file003.mpg 


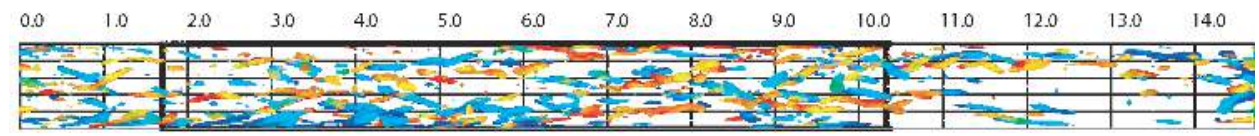

Figure 19. Instantaneous isosurfaces of $Q=0.7 U_{h}^{2} / D_{h}^{2}$, colored by the longitudinal vorticity, close to the concave wall: $R_{c}=10.5 D_{h}$, see http://www.informaworld.com/mpp/uploads/jot-2005-0072-file004.mpg

(see Patel and Sotiropoulos [12]). In the concave wall boundary layer, the comparison with the experiments shows a good agreement for the case $R_{c}=10.5 D_{h}$. For the smaller curvature radii $6.5 D_{h}$ and $3.5 D_{h}$, the velocity becomes inferior to the experimental data for $n^{+}$situated between 10 and 100. Furtheraway from the wall, all the curves superpose. Figures 15(c) and (d), respectively, correspond with $\theta=30^{\circ}$ and $\theta=45^{\circ}$. As previously shown, the intensity of the Ekman cells is important at these stations and both the longitudinal pressure gradient and the cells play a significant role in the modification of the streamwise velocity profile. These combined effects lead to a quasi-disappearance of the log-law in the convex boundary layer. A log-law can still be observed in the case with a large curvature radius $\left(R_{c}=10.5 D_{h}\right)$, but it becomes shorter and shorter when the Ekman cells intensify as we move from $\theta=30^{\circ}$ to $\theta=45^{\circ}$. It is interesting to note the good qualitative agreement between our results and the wall-law predictions in curved boundary layers based on the mixing-length analysis (see section 3.2 of Patel and Sotiropoulos [12]): for the convex curvature, the velocity profile lies above the log-law and this overshoot is more and more pronounced for an increasing curvature. In contrast, for the concave curvature, the velocity profile lies below the log profile with a deviation with is growing for an increasing curvature radius. The reader is referred to figure 3 of Patel and Sotiropoulos [12]. A good agreement between the numerical results and the experimental data is observed for the case $R_{c}=10.5 D_{h}$ except near the duct core for $\theta=45^{\circ}$.

3.2.2 Coherent vortices. Piomelli et al. [25] showed that a sufficient acceleration associated with a favourable pressure gradient induces a significant modification of the instantaneous coherent vortices of the boundary layer: these become fewer and more elongated in the streamwise direction. We now concentrate on the vortices present in the boundary layers of the curved duct. To visualize these vortices, we use the $Q$ criterion: we remind that positive $Q$ regions are associated with intense coherent vortices, [27, 28]. $Q$ is defined as

$$
Q=\frac{1}{2}\left(\Omega_{i j} \Omega_{i j}-S_{i j} S_{i j}\right),
$$

where $\Omega_{i j}$ and $S_{i j}$ are, respectively, the antisymmetric and the symmetric parts of the velocity gradient tensor. In figures 16-21 and animations 1 to 6, we show $Q$ isosurfaces coloured by the longitudinal vorticity close to each curved walls: the vortices are seen from above the considered wall. The rectangles in thick-lines indicate the curved part of the duct. The three different values of the curvature radius are here considered. As previously shown, on

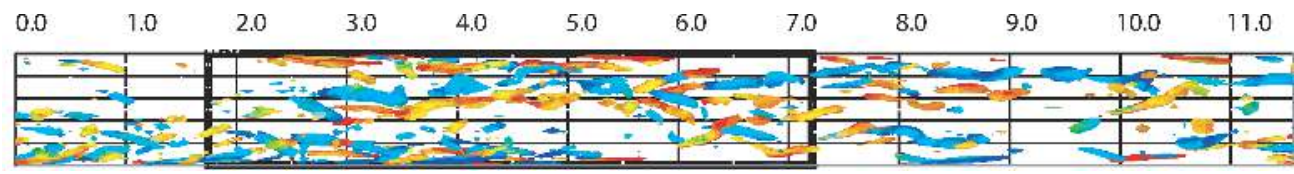

Figure 20. Instantaneous isosurfaces of $Q=0.7 U_{h}^{2} / D_{h}^{2}$, colored by the longitudinal vorticity, close to the concave wall: $R_{c}=6.5 D_{h}$, see http://www.informaworld.com/mpp/uploads/jot-2005-0072-file005.mpg 


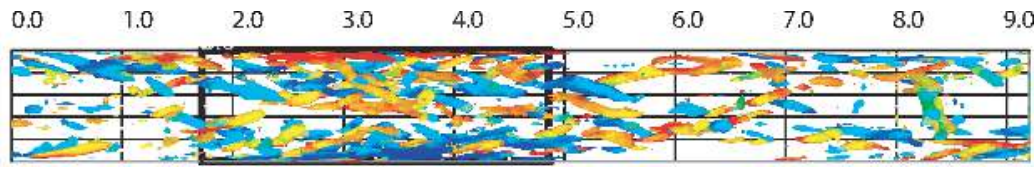

Figure 21. Instantaneous isosurfaces of $Q=0.7 U_{h}^{2} / D_{h}^{2}$, colored by the longitudinal vorticity, close to the concave wall: $R_{c}=3.5 D_{h}$, see http://www.informaworld.com/mpp/uploads/jot-2005-0072-file006.mpg

the convex wall, the streamwise pressure gradient is at first negative (favourable pressure gradient) which signifies an acceleration in the boundary layer and a stabilizing action on the flow. Consequently, we observe in figures 16-18 and animations $1-3$, that the quasilongitudinal vortices issued from the straight inlet become less numerous from the beginning of the curved part $\left(s / D_{h}=1.7\right)$. Then new vortices develop from the middle of the curved part: these are concentrated within the core of the two Ekman cells and we see that each cell is constituted of longitudinal vortices whose longitudinal vorticity has a fixed sign. We observe that the vortices concentration is more and more pronounced for smaller and smaller curvature radii.

Close to the opposite wall, concave wall, figures 19-21 and animations 4-6, the streamwise pressure gradient is positive (adverse) near the beginning of the curved part (see figure 14): these lead to more numerous vortical structures. When the curvature radius is decreased, the size of the vortices increases and these are less elongated: it is directly connected with the larger value of the positive streamwise pressure gradient in that case. At the end of the curved part, we observe that for $R_{c}=3.5 D_{h}$ the change in the sign of the pressure gradient induces a rarefaction of the structures. The notable increase in the number of vortices in the curved part is attributable to the destabilizing effect of the concave curvature associated with the centrifugal instability. The instability is more and more pronounced when the curvature radius is decreased.

We next propose to quantify the previous observations related to the variation of coherent vortices concentration with varying the curvature radius. We chose to identify the presence of coherent vortices through flow regions where $Q$ exceeds a given threshold. According to the previous studies related to the wall bounded flows, we estimate that $Q=0.6 U_{b}^{2} / D_{h}^{2}$ is a well-adapted threshold, see [28]. We then plot, in figure 22, the percentage of points where this threshold is overtaken in each downstream sections and for the three values of the curvature

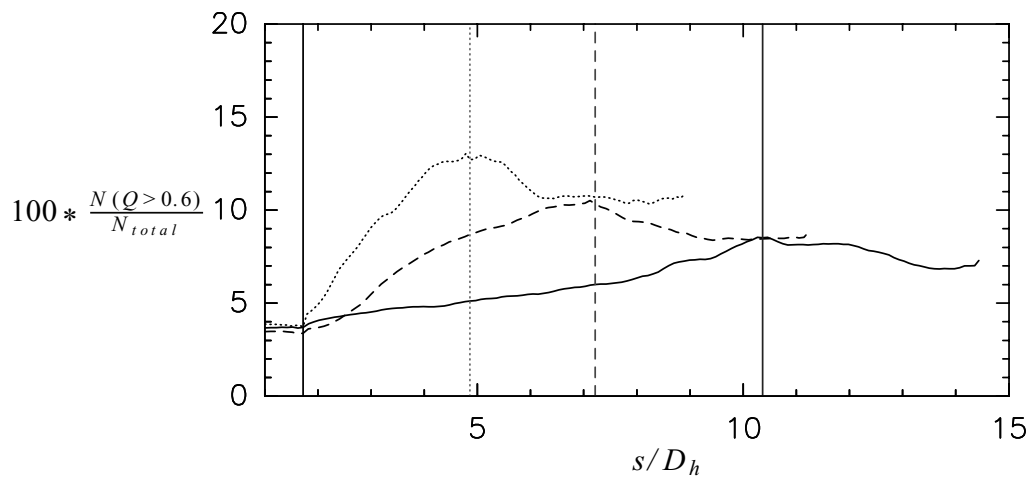

Figure 22. Percentage of points for which $Q>0.6 U_{b}^{2} / D_{h}^{2}: 100 * N(Q>0.6) / N_{\text {total }}\left(N_{\text {total }}\right.$ total number of discretization points in a section) as a function of $s / D_{h}$ for : $-R_{c}=10.5 D_{h},-\cdots R_{c}=6.5 D_{h}$ and $\cdots \cdots R_{c}=3.5 D_{h}$. The vertical lines correspond to the beginning and the end of the curved parts. 

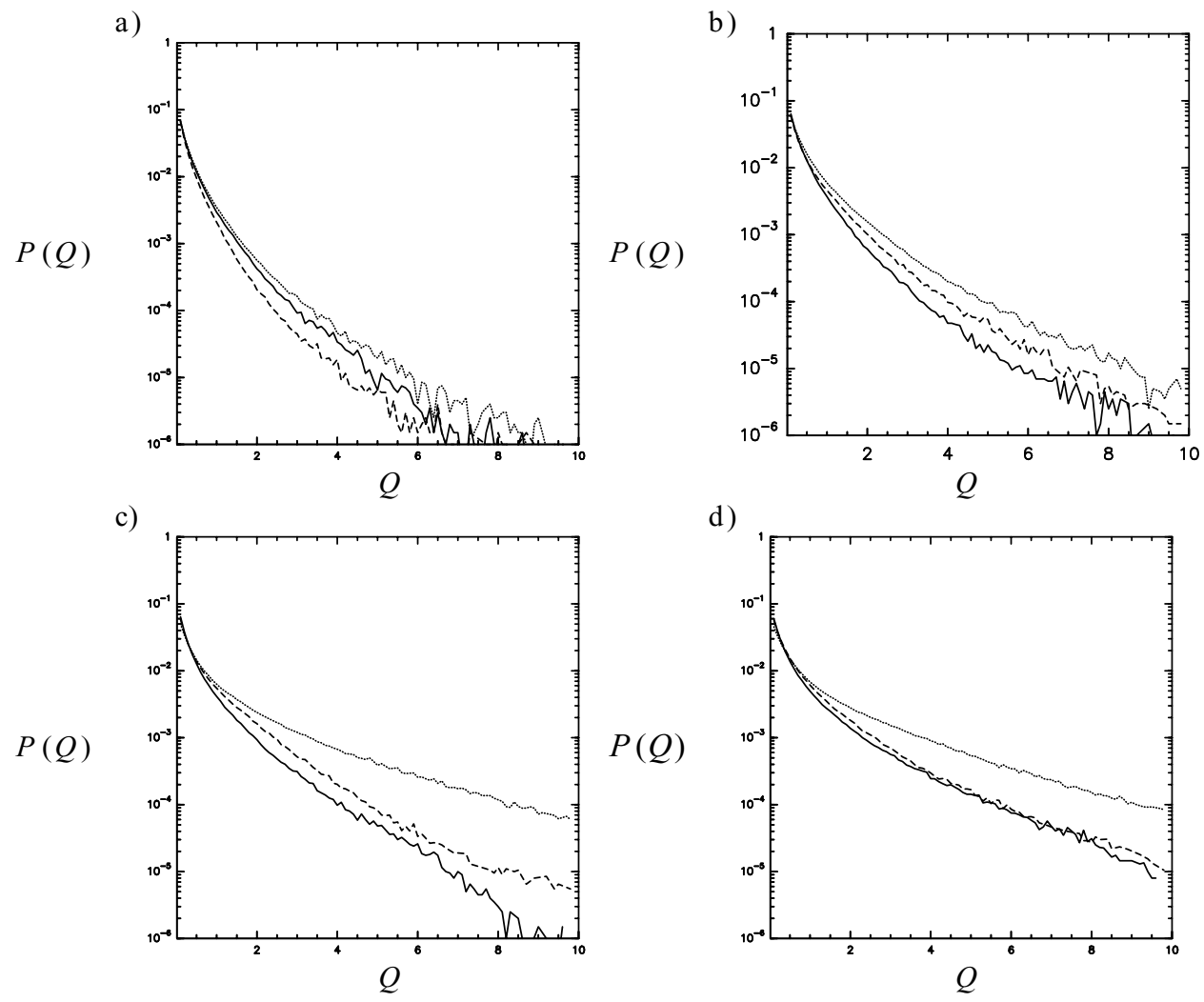

Figure 23. Normalized pdf of $Q$ for $-R_{c}=10.5 D_{h},--R_{c}=6.5 D_{h}$ and $\cdots \cdots R_{c}=3.5 D_{h}$. (a) at $s / D_{h}=1$, (b) $\theta=15$, (c) $\theta=30$ and (d) $\theta=45$.

radius. These data are obtained through a time averaging over a time period of $60 D_{h} / U_{b}$. At the inlet, the vortical structures represent only $4 \%$ of the cross-section for the three values of the curvature radius. In the curved part, an increase of the vortical structures density is observed in the three cases. The maximum vortical concentration is reached at the end of the curved part and significantly increases for small curvature radius: the peak is indeed of about $12 \%$ for $R_{c}=3.5 D_{h}$. This confirms the observations of figures 16-21. Subsequently, in the straight outflow, the vortices percentage decreases in the three cases but it remains higher than the percentage at the inlet: this is attributable to the long lived structures associated with the Ekman vortices.

A more detailed information about the $Q$ spatial distribution is provided by the probability density function of $Q$ in various flow sections. Figure 23 plots the normalized pdf $P(Q)$ as a function of $Q$ in four cross-sections and for the three values of $R_{c}$.

In the straight inflow, the three curves slightly differ probably due to an insufficient length of the statistical sampling. Further downstream, figures 23(b-d) clearly show the influence of the curvature radius. For large values of $Q$, the number of points corresponding to values between $Q$ and $Q+d Q$ is much larger when $R_{c}$ decreases: this confirms the higher vortical concentration for small radii. Figure 23 furthermore shows that the high $Q$ regions are more numerous but also that the $Q$ maximum is much higher for $R_{c}=3.5 D_{h}$ indicating a stronger vorticity amplification in that case. 


\section{Conclusion}

Large Eddy Simulations (LES) are carried out to determine the influence of the curvature radius, $R_{c}$, on a fully developed turbulent flow in a curved duct: three values of $R_{c}$ are thus considered: $3.5,6.5$ and 10.5 hydraulic diameters. We first show the ability of the LES to correctly reproduce the curvature effects since our simulations exhibit a good agreement with the experimental observations. We find that a diminution of $R_{c}$ induces a strong intensification of the secondary flow transverse to the bulk velocity. This can be accredited to the increase of the radial pressure gradient which is shown to drive these secondary flows. We observe that the secondary flow organizes into Ekman cells which are found to be more intense and larger in size for smaller curvature radius. We also investigate the influence of $R_{c}$ on the streamwise velocity profiles in both curved boundary layers. At the beginning of the curved part, an adverse streamwise pressure gradient develops on the concave wall which induces a diminution of $u^{+}$and a logarithmic behaviour lower than the classical one for a flat plate. The opposite effect arises on the convex side. Further downstream, the combined effects of the longitudinal pressure gradient of the Ekman cells induces a strong deviation from a logarithmic behaviour on the convex wall.

A well defined logarithmic law is observed on the concave boundary layer but only for the large value of $R_{c}$. This absence of logarithmic behaviour is of importance for near wall modelling which is often based on the existence of a logarithmic law.

As far as instantaneous coherent vortices are concerned, we first observe their rarefaction close to the convex wall, due to the favourable pressure gradient in agreement with Piomelli et al. [25] results. Further downstream, the longitudinal vortices concentrate within the core of the two counter-rotating Ekman cells. On the opposite concave wall, the flow destabilization associated with the centrifugal instability leads to a significant increase of the number of longitudinal vortices. The longitudinal pressure gradient is also shown to play a role in the size and the number of these vortices: an adverse pressure gradient being associated with their shortening and an increase in their number.

\section{Acknowledgements}

Some of the computations were carried out at the IDRIS (Institut du Développement et de Ressources en Informatique Scientifique, Paris). This work was supported by the CNES (Centre National d'Etudes Spatiales).

\section{References}

[1] Chang, S.M., Humphrey, J.A.C. and Modavi, A., 1983, Turbulent flow in a strongly curved U-bend and Downstream tangent of square cross-sections. PhysicoChemical Hydrodynamics, 4(3), 243-269.

[2] Kim, W.J. and Patel, V.C., 1994, Origin and decay of longitudinal vortices in developing flow in a curved rectangular duct. Journal of Fluid Engineering, 116, 45-52.

[3] Humphrey, J.A.C., Whitelaw, J.H. and Yee, G., 1981, Turbulent flow in square duct of strong curvature. Journal of Fluid Mechanics, 103, 443-463.

[4] Hunt, I.A. and Joubert, P.N., 1979, Effects of small streamline curvature on turbulent duct flow. Journal of Fluid Mechanics, 91(4), 633-659.

[5] Mees, P.A.J., Nandakumar, K. and Masliyah, J.H., 1996, Secondary instability of flow in a curved duct of square cross-section. Journal of Fluid Mechanics, 323, 387-409.

[6] So, R.M.C. and Mellor, G.L., 1975, Experiment on turbulent boundary layers on a concave wall. Aeronautical Quarterly, 26, 25-40.

[7] Moser, R.D. and Moin, P., 1987, The effects of curvature in wall-bounded turbulent flows. Journal of Fluid Mechanics, 175, 479-510. 
[8] Lund, T.S. and Moin, P., 1996, Large-eddy-simulation of a concave wall boundary layer. International Journal of Heat and Fluid Flow, 17(3), 290-295.

[9] Gillis, J.C. and Johnston, J.P., 1983, Turbulent boundary layer flow and structure on a convex wall and its redevelopment on a flat wall. Journal of Fluid Mechanics, 135, 123-153.

[10] Hoffman, P.H., Muck, K.C. and Bradshaw, P., 1985, The effect of concave surface curvature on turbulent boundary layers. Journal of Fluid Mechanics, 161, 371-403.

[11] Silva Lopes, A. and Piomelli, U., 2003, Large eddy simulation of the flow in S-duct. AIAA Journal, 964, 1-14.

[12] Patel, V.C. and Sotiropoulos, F., 1997, Longitudinal curvature effects in turbulent boundary layers. Progress in Aerospace Sciences, 33, 1-70.

[13] Bradshaw, P., 1987, Turbulent secondary flows. Annual Review of Fluid Mechanics, 19, 53-74.

[14] Paik, J., Ge, L. and Sotiropoulos, F., 2004, Toward the simulation of complex 3D shear flows using unsteady statistical turbulence models. International Journal of Heat and Fluid Flow, 25, 513-527.

[15] Johnson, P., 1990, PhD Thesis, Stanford University.

[16] Salinas Vasquez, M. and Métais, O., 2002, Large eddy simulation of turbulent flow through a heated square duct. Journal of Fluid Mechanics, 453, 201-238.

[17] Hébrard, J., Salinas Vasquez, M. and Métais, O., 2005, The spatial development of turbulent flow within a heated duct. Journal of Turbulence, $\mathbf{6}$.

[18] Hébrard, J., Métais, O. and Salinas Vasquez M., 2004, Large-eddy simulation of turbulent duct flow: heating and curvature effects. International Journal of Heat and Fluid Flow, 25, 569-580.

[19] Lesieur, M. and Métais, O., 1996, New trends in large eddy simulation of turbulence. Annual Review of Fluid Mechanics, 28, 45-82.

[20] Kennedy, C.A. and Carpenter, M.H., 1997, Comparison of several numerical Methods for simulation of compressible shear layers. NASA Technical Paper, 3484.

[21] Münch, C. and Métais, O., 2005, Large eddy simulation of turbulent flow in heated curved ducts: influence of the Reynolds number. TSFP4 Williamsburg, VA, USA.

[22] Poinsot, T.J. and Lele, S.K., 1992, Boundary conditions for direct simulations of compressible viscous flows. Journal of Computational Physics, 101, 104-129.

[23] Münch, C. and Métais O., 2005, Turbulence in cooling channels of rocket engines: Large eddy simulation. Computes Rendus Mecanique, 333, 574-579.

[24] Sotiropoulos, F. and Patel, V.C., 1993, Evaluation of some near-wall models for the Reynolds-stress transport equations in a complex 3-D shear flow. Near-Wall Turbulent Flows (Amsterdem: Elsevier), 987-997.

[25] Piomelli, U., Balaras, E. and Pascarelli, A., 2000, Turbulent structures in accelerating boundary layers. Journal of Turbulence, 1.

[26] Gavrilakis, S., 1992, Numerical simulation of low-Reynolds-number turbulent flow trough a straight square duct. Journal of Fluid Mechanics, 244, 101-129.

[27] Hunt, J., Wray, A. and Moin, P., 1988, Eddies, streams, and convergence zones in turbulent flows. Center for Turbulent Research, 193.

[28] Dubief, Y. and Delcayre, F., 2000, On coherent-vortex identification in turbulence. Journal of Turbulence, 1. 